\title{
Cognitive Influences in Parkinson's Disease Patients and Their Caregivers: Perspectives From an Australian Cohort
}

\author{
Michal Lubomski ${ }^{1,2,3 *}$, Ryan L. Davis ${ }^{2 t \neq}$ and Carolyn M. Sue ${ }^{1,2+\neq}$ \\ ${ }^{1}$ Department of Neurology, Royal North Shore Hospital, Northern Sydney Local Health District, St Leonards, NSW, Australia, \\ ${ }^{2}$ Department of Neurogenetics, Kolling Institute, Faculty of Medicine and Health, University of Sydney, Northern Sydney \\ Local Health District, St Leonards, NSW, Australia, ${ }^{3}$ School of Medicine, The University of Notre Dame Australia, Sydney, \\ NSW, Australia
}

OPEN ACCESS

Edited by:

Huifang Shang,

Sichuan University, China

Reviewed by:

Tien K. Khoo,

Griffith University, Australia

Moran Gilat,

KU Leuven, Belgium

*Correspondence:

Michal Lubomsk

mlub6241@uni.sydney.edu.au

${ }^{\dagger}$ These authors share last authorship

¥ORCID:

Michal Lubomsk orcid.org/0000-0003-4990-9293

Ryan L. Davis orcid.org/0000-0003-0512-8989

Carolyn M. Sue

orcid.org/0000-0003-1255-3617

Specialty section:

This article was submitted to

Movement Disorders,

a section of the journal

Frontiers in Neurology

Received: 28 February 2021 Accepted: 11 October 2021 Published: 15 November 2021

Citation:

Lubomski M, Davis RL and Sue CM

(2021) Cognitive Influences in

Parkinson's Disease Patients and

Their Caregivers: Perspectives From

an Australian Cohort.

Front. Neurol. 12:673816

doi: 10.3389/fneur.2021.673816
Objectives: Cognitive impairment impacts negatively on Parkinson's disease (PD) patient and caregiver quality of life (QoL). We examined cognitive impairment in PD patients and their caregivers to determine if caregiver cognition affected their PD relative.

Methods: Validated cognition and clinical outcome measures were assessed in $103 \mathrm{PD}$ patients and 81 caregivers.

Results: PD patients showed more cognitive impairment than their carers, with $48.6 \%$ having possible Mild Cognitive Impairment (MCl) and 16.5\% having PD dementia. Increasing age, male gender, lower education level, various non-motor symptoms and certain therapies, associated with poorer cognition in PD. Eighteen and a half percent of caregivers were found to have $\mathrm{MCl}$, in association with a lower physical and mental QoL. This reflected in lower QoL and mood for the respective PD patients.

Conclusion: Impaired cognition and QoL in caregivers was associated with decreased QoL and mood for respective PD patients, suggesting $\mathrm{MCl}$ in caregivers is an important consideration for the management of PD.

Keywords: Parkinson's disease, cognition, caregiver, cognitive impairment, dementia

\section{INTRODUCTION}

Parkinson's disease (PD) is a progressive multisystem disorder that contributes to significant morbidity, healthcare and caregiver burden $(1,2)$. Cognitive impairment can be present in as many as $30 \%$ of PD patients at disease onset, progressing to PD Dementia (PDD) in up to $80 \%$ of patients with advanced disease (3). The concept of mild cognitive impairment (MCI) is an intermediate clinical state between normal cognitive ageing and dementia (4). MCI represents an important window in time where patients are still functional in their day to day life but are at a higher risk of developing PDD (5). Despite a growth in literature evaluating cognitive changes in PD patients $(3,6-8)$, there is a lack of understanding of the differences in cognition between PD patients and their caregivers $(9,10)$.

It has been suggested that $\mathrm{PD}$ caregivers experience a higher burden of care leading to reduced quality of life (QoL) with the onset of dementia in their PD relative (11), influencing PD management and delaying formal care or nursing home placement (12). As there is limited evidence 
around the influence of PD caregivers with cognitive impairment and their capacity to care for a PD patient, understanding these aspects could inform best clinical practise to improve caregiver well-being for better support and care of PD relatives.

Individuals with PDD commonly report deficits in attention, language, memory and visuospatial orientation (13). Additionally, deficits in executive functioning affect daily tasks, such as driving and medication adherence, whilst other individuals show a reduced awareness of their executive deficits and overestimate their capabilities (13). These impacts are likely to negatively influence caregiver QoL and further add to caregiver burden. Several prior studies have evaluated PD-related risk factors for cognitive impairment, highlighting potentially modifiable specific risk interventions $(14,15)$. Equally, non-modifiable factors, such as age, age at diagnosis, rigid-akinetic phenotype, physical impairment, impairment of semantic fluency, genetic factors, low education level and postural instability have also been proposed as important risk factors for PDD (15).

We hypothesised that a greater degree of cognitive impairment in PD patients would result in perceptibly lower QoL and enhancement of non-motor symptoms (NMS). Additionally, we hypothesised that the presence of cognitive impairment in PD caregivers results in a worsening of their QoL, which could potentially impact on their ability to effectively care for their affected PD relative. The study presented herein provides new insight into PD caregiver cognitive impairment and the potential impact on $\mathrm{PD}$ patient care and well-being.

\section{METHODS}

\section{Study Settings and Subjects}

Subjects were recruited between June 2018-2019 from the movement disorder and neurology clinics at Royal North Shore Hospital, Sydney, Australia, as reported in our previous study (16). Consecutive PD patients were recruited if they were $>18$ years of age, and had a clinical diagnosis of idiopathic PD according to the UK Parkinson's Disease Society Brain Bank Diagnostic Criteria (17). Caregivers were recruited if they were $>18$ years of age, they exhibited no clinical indication of PD, they had no known diagnosis of a neurodegenerative disorder, and were a spouse, sibling or child residing in the same abode. Caregivers were chosen as the comparator group due to their availability and relative matched age to PD patients. Exclusion criteria included secondary Parkinsonism and medical or surgical disorders preventing completion of questionnaires. Patients were required to read and complete the questionnaires in English language, which resulted in the exclusion of $1 \mathrm{PD}$ participant. Existing cognitive impairment, motor symptom limitation, mental illness or traumatic brain injury were not considered exclusion criteria. All PD patients and caregivers were specifically asked to complete their own questionnaires.

\section{Data Collection}

Data was collected from validated self-administered questionnaires, in addition to data on socio-demographic, lifestyle and clinical management factors (16). Both PD patients and caregivers completed the same questionnaires described below, unless the questionnaire was PD-specific (indicated by $\left.{ }^{*}\right)$. The Montreal Cognitive Assessment (MoCA) (18) has been validated as the most appropriate screening instrument for the detection of MCI and PDD (5). It is also endorsed by the International Parkinson and Movement Disorder Society (MDS) Task Force for the diagnosis of PD-MCI as being most suitable for demonstrating global cognitive deficits in a clinical setting (19). The MoCA was utilised to screen for MCI (score $<26 / 30)(5,18,20)$ and PDD (score of $<21 / 30$ and loss of one or more instrumental activity of daily living) (20). Level 1 MDS criteria were used to assess for possible MCI (21). One consultant neurologist investigator (ML) conducted all MoCA assessments with PD patients and caregivers separately, to minimise bias. The Short Form Health Survey (SF-36) (22) was used to assess QoL for both PD patients and their caregivers to examine perceived health status. Two aggregate summary scores, the Physical Component Summary (PCS) and the Mental Component Summary (MCS) were derived from the eight concepts. Each scale was scored from 0 (most disability) to 100 (least disability). PD patients also completed the PDQ-39

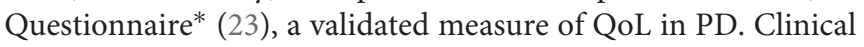
depression was determined by the Beck Depression Inventory (BDI), with scores ranging 0-63 (24). Depression was diagnosed in accordance with validated BDI cut-off criteria for PD patients $(>13)$ and caregivers $(>9)(25,26)$.

A number of other questionnaires were used to assess nonmotor symptoms in the PD cohort (27). Constipation severity was evaluated by the Rome-IV criteria (28) and the Cleveland Constipation Score (CCS) (29). Physical activity was assessed by the International Physical Activity Questionnaire (IPAQ) (30) and NMS were assessed by the Non-Motor Symptoms Scale* (NMSS), scored between 0 (least affected) to 243 (most affected) (31). Clinical motor assessments were performed by a neurologist during the patient's 'on' state, as a measure of the existing motor function, in accordance with the International Parkinson and Movement Disorder Society—Unified Parkinson's Disease Rating Scale-Part III (MDS-UPDRS III) criteria* (32). PD phenotype was determined from MDS guidelines (33). PD medications were compared following standard methods for calculating daily levodopa equivalent dose (LED)* (34), whilst Impulse Control Disorder (ICD)* was defined according to established diagnostic criteria (35).

\section{Statistical Analysis}

Normal distribution of all data was confirmed using the Shapiro-Wilk test. Two-sample, independent $t$-tests were used to analyse differences between the groups for continuous variables. Chi-squared tests were used to compare differences between categorical variables. Logistic and linear regression models were constructed to evaluate differences in the prevalence of cognitive features between the PD and caregiver groups, as well as within the PD cohort, after controlling for demographic and clinical variables. Pearson correlations were used to evaluate relationships between clinically relevant variables. $p<0.05$ was set as the level of statistical significance. Data analysis was performed using SPSS, version 26 (SPSS Inc, Chicago, IL, USA). 
TABLE 1 | Cohort demographic and clinical characteristics.

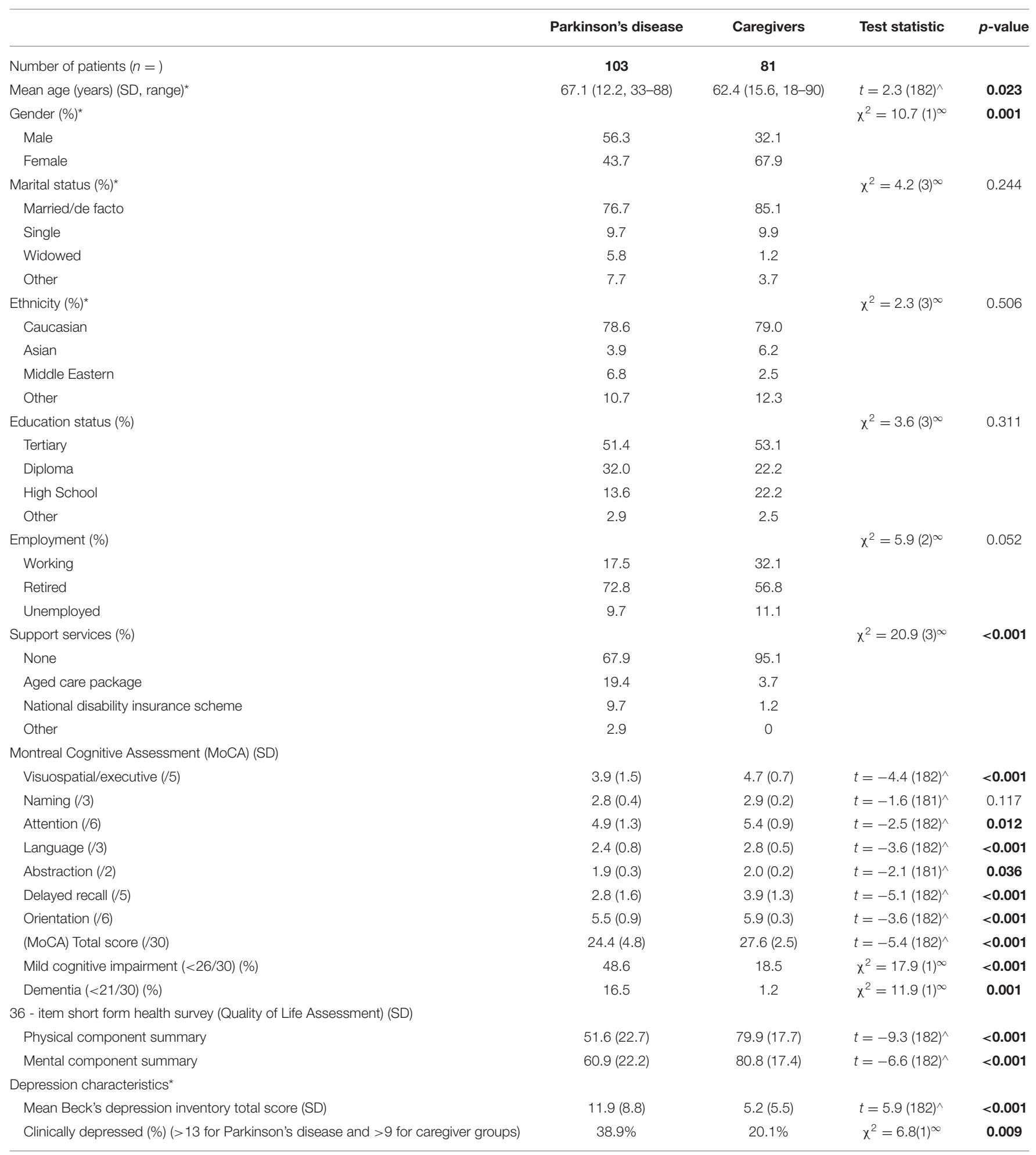

$\wedge$ Independent sample t-test.

Pearson's chi-squared test.

"This data is partially reproduced from Lubomski et al. (16) and Lubomski et al. (36).

df, degrees of freedom; $S D$, Standard Deviation.

Bold $p$-values are statistically significant at $p<0.05$. 


\section{RESULTS}

\section{Demographic Characteristics}

Demographic information pertaining to the cohort studied here has been reported previously (16). In summary, 103 PD patients, mean age 67.1 years [Standard Deviation (SD 12.2)], 56.3\% male and 81 caregivers, mean age 62.4 years (SD 15.6), 32.1\% male were recruited (Table 1). Within the PD cohort, the mean age of symptom onset was 58.8 years (SD 13.6) and mean duration of disease was 9.2 years (SD 6.5). Half of the PD patients reported a late disease onset ( $>60$ years), whilst $11 \%$ had early onset $(<40$ years) (Table 2). Of the NMS, half of the PD patients reported Rapid Eye Movement Sleep Behaviour Disorder (RBD), threequarters reported hyposmia and one fifth identified having an impulse control disorder (ICD). In terms of risk factors for cognitive impairment, $18.4 \%$ had a family history of PD, $10.7 \%$ reported a significant history of head trauma, and $2.9 \%$ had previously used neuroleptic medication. Approximately 5\% of the PD cohort was treatment naïve, whilst those on treatment regimens had a mean daily LED of $834.8 \mathrm{mg}$ (SD 527.3). The mean MDS-UPDRS III score was 32.9 (SD 17.9). Utilisation of standard and device assisted therapies, the frequency and severity of gastrointestinal symptoms, chronic pain, physical activity and NMS in the PD cohort are further outlined in Table 2.

\section{Clinical Characteristics Cognitive Differences Between Parkinson's Disease Patients and Their Caregivers}

PD patients showed significantly decreased cognition scores compared to their caregivers, across six out of the seven subscores assessed by the MoCA, with the only comparable section being "naming" (all $p<0.05$; Table 1). The mean total MoCA score (MoCA TS) in the PD cohort was 24.4 [(SD 4.8), range 9-30], compared to the caregiver group, 27.6 [(SD 2.5), range $18-30, p<0.001$ ]. $48.6 \%$ of PD patients vs. $18.5 \%$ of caregivers met the criteria for possible MCI $(p<0.001)$, whilst $16.5 \%$ of the PD cohort met the criteria for PDD and $1.2 \%$ of the caregiver group for dementia (MoCA TS $<21 / 30$ and loss of one or more instrumental activities of daily living; Table 1) (20). This indicates that cognition is considerably lower in PD patients compared to their caregivers, accounting for disease-associated differences.

Associations between impaired cognition and a poorer QoL were identified when combining the PD patient and caregiver groups (combined cohort), with correlations between the MoCA TS and the SF-36 PCS $(r=0.423, p<0.001)$ and MCS scores $(r=0.352, p<0.001)$, respectively. Further, individuals in the combined cohort who were identified to have MCI, tended to have lower PCS $(r=0.335, p<0.001)$ and MCS scores $(r=$ $0.309, p<0.001)$. Assessing QoL associations specifically within the caregiver group revealed correlations between the MoCA TS with the SF-36 PCS $(r=0.301, p=0.006)$ and MCS scores $(r=0.289, p=0.033)$. Consistently, caregivers with MCI had lower PCS $(r=0.277, p=0.044)$ and MCS scores $(r=0.264$, $p=0.047$ ), signifying a lower QoL if cognitive impairment was present. Logistic regression models evaluating cognitive differences between the groups showed statistical significance for both the MoCA TS (Wald $\chi^{2}=21.1, p<0.001$ ) and the
TABLE 2 | Parkinson's disease clinical characteristics.

\begin{tabular}{|c|c|}
\hline Mean age at diagnosis (years) (SD, range) ${ }^{\star}$ & $58.8(13.6,24-88)$ \\
\hline Mean Parkinson's disease duration (years) (SD, range) ${ }^{\star}$ & $9.2(6.5,1-30)$ \\
\hline \multicolumn{2}{|l|}{ Parkinson's disease phenotype $(\%)^{\star}$} \\
\hline Tremor dominant & 30.1 \\
\hline Postural instability and gait impairment & 20.4 \\
\hline Akinetic rigid & 38.9 \\
\hline Young onset ( $<40$ years) & 10.7 \\
\hline Late onset (>60 years) & 49.5 \\
\hline \multicolumn{2}{|l|}{ Disease complication (\%)* } \\
\hline Motor fluctuations & 58.3 \\
\hline Dyskinesia & 58.3 \\
\hline Wearing off & 81.6 \\
\hline Impulse control disorder & 19.4 \\
\hline \multicolumn{2}{|l|}{ Non-motor symptom (\%) } \\
\hline Hyposmia & 73.8 \\
\hline REM sleep behaviour disorder & 48.5 \\
\hline \multicolumn{2}{|l|}{ Risk factor (\%) } \\
\hline Family history of Parkinson's disease & 18.4 \\
\hline Prior neuroleptic use & 2.9 \\
\hline Any head trauma & 10.7 \\
\hline Levodopa equivalent daily dose (mg), (SD, range) ${ }^{\star}$ & $\begin{array}{c}834.8(527.3 \\
0-2,186)\end{array}$ \\
\hline $\begin{array}{l}\text { Mean MDS unified Parkinson's disease rating scale-III } \\
\text { ('on' state) (SD, range) }{ }^{\star}\end{array}$ & $32.9(17.7,5-91)$ \\
\hline \multicolumn{2}{|l|}{ Parkinson's disease therapy $(\%)^{\star}$} \\
\hline Treatment naïve & 4.9 \\
\hline Oral levodopa & 89.3 \\
\hline Dopamine agonist & 40.0 \\
\hline Monoamine oxidase B inhibitor & 18.4 \\
\hline Anticholinergic & 12.6 \\
\hline Catechol-O-methyl transferase inhibitor & 23.3 \\
\hline Amantadine $^{\#}$ & 12.6 \\
\hline Levodopa/carbidopa intestinal gel & 8.7 \\
\hline Deep brain stimulation & 10.7 \\
\hline Apomorphine (subcutaneous infusion) & 6.8 \\
\hline \multicolumn{2}{|l|}{ Quality of life } \\
\hline PDQ-39 summary index (SD) & $29.2(17.3)$ \\
\hline MDS non-motor symptoms score (NMSS) - total score (SD) & $62.7(42.9)$ \\
\hline \multicolumn{2}{|l|}{ Gastrointestinal symptoms ${ }^{\star}$} \\
\hline Cleveland constipation score (SD) & $7.2(4.7)$ \\
\hline Constipation score as per ROME IV criteria (SD) & $4.4(3.5)$ \\
\hline Functional constipation as per ROME IV criteria (\%) & 78.6 \\
\hline Chronic pain over last 3 months (\%)* & 72.8 \\
\hline Pain score (visual analogue scale, 0-10) (SD) & $4.9(2.5)$ \\
\hline International physical activity questionnaire (IPAQ) score & $1823.6(1693.6)$ \\
\hline
\end{tabular}

(MET-minutes/week) (SD)*

$S D$, Standard Deviation.

\#Not considered an anticholinergic agent.

*This data is partially reproduced from Lubomski et al. (16).

proportion of individuals with MCI (Wald $\chi^{2}=16.8, p<$ $0.001)$. Statistical significance persisted between these groups when controlling for age, sex, QoL (PCS and MCS), depression 
(BDI score) and constipation (Rome-IV criteria), MoCA TS (Wald $\chi^{2}=3.8, \mathrm{df}=5, p=0.044$ ) and MCI (Wald $\chi^{2}=3.7$, $\mathrm{df}=5, p=0.048)$, respectively.

\section{Cognition in Parkinson's Disease}

Within the PD and caregiver cohorts, linear regression models showed a decline in cognitive function with increasing age, when controlling for sex and PD duration $\left(\beta=-0.303, r^{2}=\right.$ 0.185, $p=0.001$ and $\beta=-0.307, r^{2}=0.101, p=0.006$ ), respectively. Significant gender differences were also identified, with male PD patients scoring a lower mean MoCA TS [23.4, (SD 5.3)] compared to PD women [25.8, (SD 3.8), $t=-2.6, p$ $=0.011]$. Linear regression modelling confirmed that men had greater cognitive impairment, corresponding to a lower MoCA TS, when controlling for age and PD duration $(\beta=0.274$, $\left.r^{2}=0.185, p=0.005\right)$. A lower level of education was also associated with increased cognitive impairment, as those who completed either a diploma or high school certificate had a lower MoCA TS compared to individuals who had completed tertiary studies, independent of age, sex and PD duration $(\beta=-0.296$, $\left.r^{2}=0.282, p=0.002\right)$. No associations between ethnicity, marital status, employment or support service utilisation and the MoCA TS were identified. Interestingly, PD individuals with $\mathrm{RBD}$ were more likely to have a lower MoCA TS compared to those without RBD, after controlling for age, sex and PD duration [23.2 (SD 5.4) vs. 25.5 (SD 3.9), $\beta=0.186, r^{2}=$ $0.196, p=0.043$ ]. No associations between cognition and PD phenotype, dyskinesia, on/off fluctuations, ICDs, anosmia, previous neuroleptic use, head trauma, or family history of PD were identified.

\section{Treatment Influences}

Individuals with increased motor severity, as well as those who reported 'wearing off' of their treatments, were noted to have a lower MoCA TS, after controlling for age, sex, PD duration and daily $\operatorname{LED}\left(\beta=-0.475, r^{2}=0.390, p<0.001 ; \beta=0.197, r^{2}=\right.$ $0.199, p=0.035$, respectively). Regarding clinical management, individuals utilising anticholinergics $\left(\beta=0.195, r^{2}=0.147, p=\right.$ $0.043)$ and apomorphine infusions $\left(\beta=0.257, r^{2}=0.226, p=\right.$ $0.005)$ were more likely to have a lower MoCA TS compared to all the other standard and advanced therapies, after controlling for age, sex, and PD duration. Despite controlling for patient age and PD duration for both of these modalities, their use may select out individuals with more refractory $\mathrm{PD}$ and who are more prone to cognitive decline. Nevertheless, individuals with PDD were also more likely to utilise apomorphine subcutaneous infusion compared to any other standard or device assisted therapy $(\beta=$ $\left.0.199, r^{2}=0.108, p=0.044\right)$ and required a higher daily LED $(\beta$ $\left.=-0.224, r^{2}=0.106, p=0.047\right)$, after controlling for age, sex, and $\mathrm{PD}$ duration.

\section{Cognitive Impacts on Quality of Life and Non-motor Symptoms}

In the PD cohort, individuals with a lower MoCA TS were noted to report a lower QoL. These scores were accompanied by correspondingly lower SF-36 PCS $\left(\beta=0.266, r^{2}=0.241, p\right.$ $=0.009)$ and MCS scores $\left(\beta=0.289, r^{2}=0.250, p=0.006\right)$, indicating increased physical and psychological disability, after controlling for age, sex and PD duration. Also, individuals who met the criteria for MCI showed poorer QoL, with lower SF36 PCS $\left(\beta=0.203, r^{2}=0.213, p=0.047\right)$ and MCS scores $(\beta$ $\left.=0.325, r^{2}=0.257, p=0.002\right)$, when controlling for age, sex, and PD duration. QoL assessed by the PDQ-39 Summary Index (PDQ-39 SI) identified that PD patients with a lower MoCA TS $\left(\beta=-0.369, r^{2}=0.377, p<0.001\right)$, who had MCI $(\beta=-0.234$, $\left.r^{2}=0.312, p=0.013\right)$ or PDD $\left(\beta=-0.252, r^{2}=0.326, p=\right.$ 0.004 ), were more likely to report a reduced QoL (i.e., higher PDQ-39 score), after controlling for age, sex and PD duration. Perhaps also not surprisingly, individuals who reported increased NMS, assessed by the NMSS, were also more likely to show worse cognitive function, with a lower MoCA TS $\left(\beta=-0.412, r^{2}=\right.$ $0.181, p<0.001)$ in conjunction with MCI $\left(\beta=-0.324, r^{2}=\right.$ $0.129, p=0.003)$ or PDD $\left(\beta=-0.266, r^{2}=0.108, p=0.009\right)$, after controlling for age, sex and $\mathrm{PD}$ duration.

Importantly, $\mathrm{PD}$ patients without $\mathrm{MCI}$ were noted to experience an even lower QoL when their caregiver had MCI, compared to a caregiver with no MCI [PCS 50.9 (SD 22.3) vs. 55.8 (SD 16.1), $t=-2.5, p=0.015$ and MCS 60.5 (SD 18.4) vs. 64.8 (SD 19.3), $t=-2.3, p=0.028$ ]. These findings were also observed utilising the PDQ-39 SI [28.2 (SD 23.2) vs. 25.9 (21.1), $t$ $=-2.1, p=0.038]$. No statistically significant differences in QoL change were noted for PD patients with $\mathrm{MCI}$ who had caregivers with or without MCI.

\section{Gastrointestinal Influences}

Gastrointestinal (GI) dysfunction was also associated with differences in cognitive function within the PD cohort. Individuals diagnosed with constipation according to the Rome-IV criteria, more often had MCI $\left(\beta=0.174, r^{2}\right.$ $=0.203, p=0.044)$, when controlling for age, sex, LED, anticholinergic medication use and PD duration. Accordingly, increasing constipation severity negatively correlated with cognitive function (i.e., lower MoCA TS) on the Rome-IV ( $r=$ $-0.238, p=0.015)$ and CCS $(r=-0.240, p=0.015)$, being most notable in individuals with PDD (Rome-IV; $r=-0.378, p=$ 0.004 and CCS; $r=-0.381, p=0.004)$.

\section{Mood Influences}

PD patients diagnosed with depression were noted to have greater cognitive impairment (i.e., lower MoCA TS; $\beta=-0.259, r^{2}$ $=0.251, p=0.004)$ and were more likely to have MCI $(\beta$ $\left.=-0.213, r^{2}=0.222, p=0.021\right)$ or $\operatorname{PDD}\left(\beta=-0.190, r^{2}\right.$ $=0.133, p=0.049)$, when controlling for age, sex and PD duration. The BDI score and MoCA TS showed a significant weak negative correlation $(r=-0.284, p=0.004)$, as well as for those individuals with a MoCA TS meeting PDD criteria $(r$ $=-0.226, p=0.022$ ). Furthermore, PD patients without MCI were noted to be more depressed when their caregiver had MCI, compared to a caregiver with no MCI [BDI 13.4 (SD 10.2) vs. 9.8 (SD 9.8), $t=-1.9, p=0.041$ ]. No statistically significant changes in depression were noted for PD patients with MCI who had caregivers with or without MCI. 


\section{Physical Exercise and Chronic Pain Influences}

$\mathrm{PD}$ patients who reported increased physical activity (metabolic equivalent-minutes/week, assessed by the IPAQ) were noted to have decreased cognitive impairment (i.e., higher MoCA TS; $\beta$ $\left.=0.204, r^{2}=0.220, p=0.040\right)$, as well as reflecting a lower likelihood of having MCI $\left(\beta=0.216, r^{2}=0.217, p=0.030\right)$ or $\operatorname{PDD}\left(\beta=0.208, r^{2}=0.114, p=0.049\right)$, when controlling for age, sex and PD duration. Correlations between the IPAQ score and the MoCA TS $(r=0.251, p=0.011)$, MCI $(r=0.246, p=0.012)$ or PDD $(r=0.221, p=0.025)$, were also identified. Furthermore, individuals who reported experiencing chronic pain were more likely to have increased cognitive impairment (i.e., lower MoCA TS; $\beta=0.187, r^{2}=0.218, p=0.044$ ), after controlling for age, sex and PD duration. Interestingly, no associations between pain severity and cognitive function were identified.

\section{DISCUSSION}

Our study identified numerous clinically relevant cognitive differences across a number of demographic and clinical characteristics in our PD patient cohort (Figure 1). In addition, several important insights into PD caregivers, including the QoL and cognition of carers that may impact PD patient care, were identified. Multiple analyses were performed (PD normal cognition vs. PD MCI vs. PD caregiver with normal cognition vs. PD caregiver with $\mathrm{MCI}$ ), with the most clinically significant results presented. Overall, PD patients showed greater cognitive impairment compared to their caregivers. Approximately half of the PD individuals and one fifth of their caregivers met the criteria for possible MCI, whilst $16.5 \%$ of PD patients met the criteria for PDD. These findings are comparably higher than in other studies $(5,20)$, and may be partially explained by our cohort's increased PD disease duration [mean $=9.2$ years post diagnosis (SD 6.5) vs. 6.3 and $\sim 7$ years in the other studies mentioned $(5,20)]$.

One of the key insights of this study was the severity of cognitive impairment in PD caregivers, as this has been seldom reported in the literature. Our findings suggest that nearly a fifth of the PD caregivers in this study met the criteria for MCI, inferring a potential challenge for those with MCI being able to care for a spouse or relative with cognitive impairment. The degree of MCI detected in our caregiver group is notable, although not formally quantified in our analysis. Caregiver related cognitive impairment is important to recognise in order to provide additional support systems and services for those caring for a relative with PD. Accordingly, clinical care should not just be restricted to the PD patient but should also be a courtesy extended to the caregivers when presenting to clinic with their respective $\mathrm{PD}$ patient.

It was also shown that PD patients without MCI, but who had a caregiver with MCI, were more likely to report a lower QoL than those PD patients whose caregiver did not have MCI. These findings further highlight the often-complex considerations for caregiver-related MCI negatively impacting on the outcomes of PD patient management. A variety of unintentional negative outcomes may arise from PD caregivers with MCI lacking insight into appropriate caregiving responsibilities, including potentially inadequate nutrition, hygiene and medication administration for the PD patient. Furthermore, issues may arise from failure to follow through with adequate medical treatments for PD and other comorbidities, and confusion or uncertainty around when to seek additional support. Such factors may explain why PD patients with caregivers who had MCI were more likely to report a lower QoL and increased depression severity. Such considerations are vital and should prompt further studies to rigorously examine the influences of cognitive function and the degree of cognitive impairment in PD caregivers. Moreover, improved understanding of how cognitive impairment in both $\mathrm{PD}$ caregivers and $\mathrm{PD}$ patients affects the ability to provide and receive care, may further inform clinical interactions with caregivers that bolster their efforts to provide physical, emotional, functional, and financial support to relatives with PD and themselves.

Within the PD cohort, several key demographic and clinical characteristics were shown to associate with poorer cognitive function. Increasing patient age, a lower education status, the presence of RBD and male gender appeared to reflect individuals with an increased propensity for cognitive impairment. Although contradictions in the divergence of cognitive symptoms between men and women are common in the literature, men appear to perform worse on multiple measures of cognition compared to women (37-39). Perhaps unsurprisingly, advancing age (a risk factor for impaired cognition in the healthy population) and lower education levels were related to poorer cognitive capacity. Completion of tertiary studies appeared to be associated with a protective effect against cognitive decline in PD, as was reported earlier (40). Furthermore, the data indicated that RBD patients had worse cognitive impairment, which has also been described previously (41). This highlights RBD as a potential red flag for cognitive impairment in PD (41), although the exact mechanisms are still poorly understood, they are in keeping with the hypothesised caudo-rostral spread of Lewy bodies, initially through the brainstem leading to RBD and later the neocortex resulting in cognitive decline (42).

Motor severity also appeared to reflect significant associations with cognitive function in PD. Individuals with higher MDSUPDRS-III and those reporting medications 'wearing off' were more likely to report a higher degree of cognitive impairment. Treatment with anticholinergics or apomorphine infusions were also associated with lower cognitive function. The following agents were considered anticholinergics; trihexyphenidyl, benztropine, orphenadrine, procyclidine or biperiden. Of the $16.5 \%$ of individuals with PDD, those requiring apomorphine infusions and a high daily LED were most vulnerable to cognitive impairment. The use of anticholinergic agents in PD is known to adversely affect cognition (43), despite another study finding no significant association in anticholinergic burden in early stage PD (44). Although apomorphine infusions are not indicated in those with severe dementia (45), it is our clinical experience that at low concentrations this adjuvant therapy can offset peak-effect dyskinesias and off-period non-motor symptoms, particularly in the older PD population who require device-assisted therapy.

When examining the PD patient and caregiver cohorts, individually and in combination, it was apparent that those individuals with more impaired cognition were more likely to 


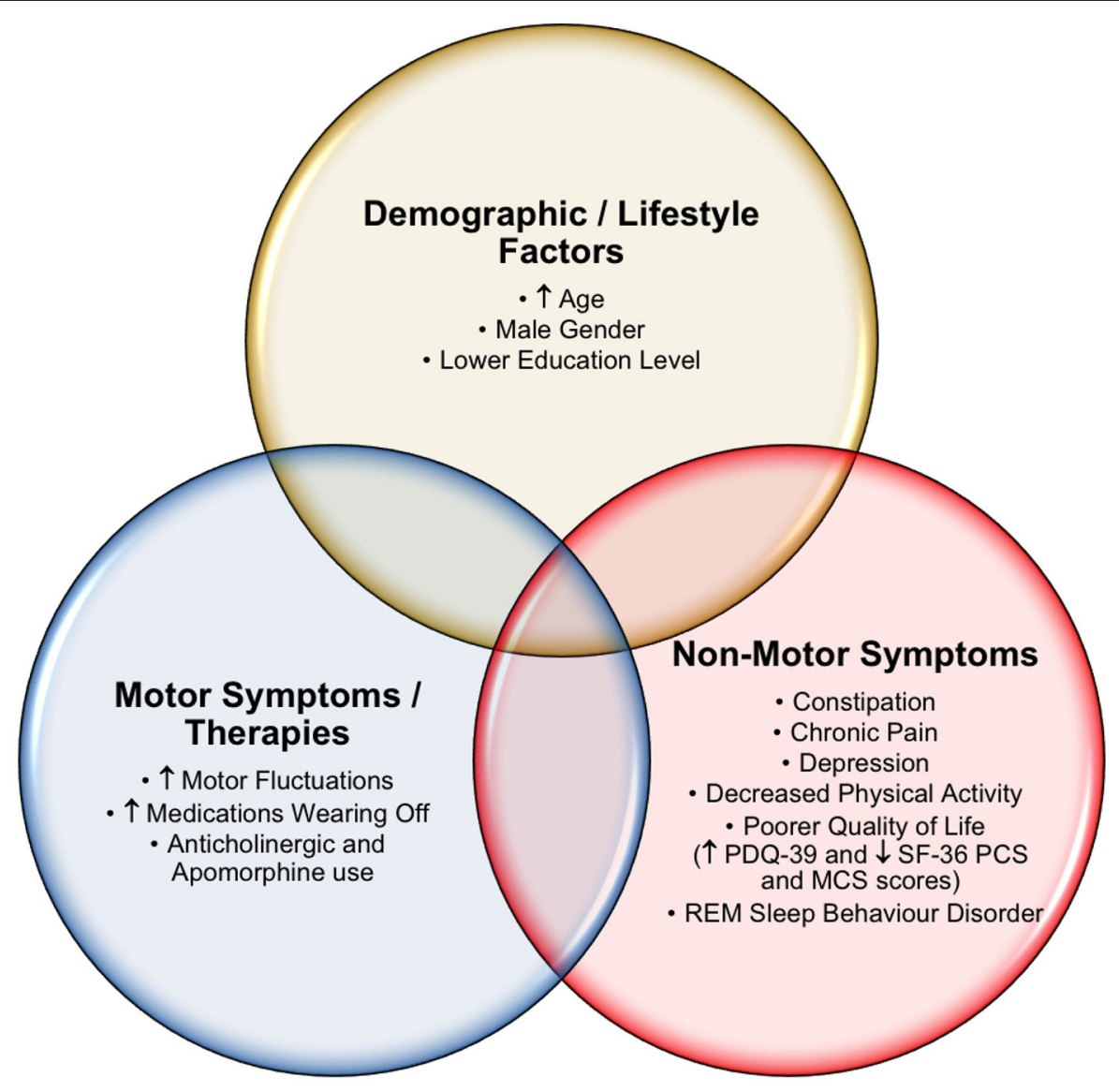

FIGURE 1 | Factors associated with impaired cognition in Parkinson's disease.

report a lower QoL, indicated by the PCS and MCS scores of the SF-36. These findings are in keeping with earlier PD studies $(46,47)$. Individualised assessments of PD patient and caregiver QoL are vital to avoid potential oversight of the impact of QoL on PD patients that are cognitively impaired, compared to many caregivers who may not be (48), as was here. Furthermore, the impacts of impaired cognition leading to a poorer QoL have been widely studied in the PD population $(47,49)$. This investigation has validated these findings, supporting the association between decreased cognitive capacity and a self-perceived reduced QoL. Anticholinesterase therapy and other non-pharmacological interventions, including cognitive rehabilitation, have been proposed as useful strategies to improve attention and concentration in order to improve patient QoL (47).

Constipation has previously been identified as a potential risk factor for cognitive impairment in PD (50). However, more recently, the ascending spread of alpha-synuclein through the microbiota-gut-brain-axis has been proposed as a link between GI dysfunction and cognitive decline (51), in addition to varying metabolic pathways of the gut microbiota that affect the efficacy of PD therapies (52). Nevertheless, within our cohort, individuals with increasing constipation severity were more likely to have poorer cognitive function, when adjusted for LED and anticholinergic medication use. Important management implications arise for treating chronic constipation in PD patients with cognitive impairment, which should focus on the institution of simplified and structured regimes, incorporating extra water and added fibre intake to their diet, in addition to routine use of aperients or laxatives to minimise risk of faecal impaction. Avoidance of unnecessary anticholinergic or opioid analgesia medication or other agents that may slow gut transit times is an important consideration and clinicians should actively enquire about their PD patient's bowel motions and GI symptoms, as a potentially modifiable risk factor for worsening cognitive function.

Lastly, this study identified that the influences of depression, chronic pain and reduced physical exercise were negatively associated with cognitive function in PD. These NMS may be challenging to manage, due to interdependent relationships with executive function, which is influenced by an individual's level of motivation, engagement and insight (53-55). Complex neurotransmitter and other neuromodulatory effects are believed to be integral in the processes leading to cognitive decline in 
PD and may be further significantly influenced by attitudes and health-related perceptions of patients and their caregivers (56). The effects of maintaining physical exercise should not be underrated as a powerful and modifiable approach to promoting improved cognition in PD (53). Our findings support increased physical activity being positively associated with cognitive function and a reduced likelihood of having MCI or PDD. Encouragement of PD patients to remain socially active, seek help if feeling depressed or anxious, adopt depression coping strategies, maintain physical exercise, eat a healthy diet and engage in cognitive training interventions have been proposed as a multidisciplinary approach to optimising NMS and cognitive impairment in PD $(36,56-59)$. These interventions would be more beneficial at the MCI stage of disease, making early identification of these patients critical.

The impacts of managing many of the PD NMS that are associated with cognitive impairment also often result in notable implications for PD caregivers. Considerations include additional need for structured and timely supervision of medications, maintaining engagement and motivation with exercise regimes, seeking extra support from friends and family, as well as other support service provision options. In addition, medical and allied health consultation requirements, excess costs associated with the provision of support services, alteration to a healthy diet and medication scheduling are also often encountered. Many of these interventions are dependent on health-related perceptions and cognitive function of the PD caregivers (56). This may lead to excess caregiver stress and potential burnout (60), which can impact on the quality, standard and vigilance of care afforded to their PD patient. Therefore, impacts on caregivers need to be actively screened for and considered to ensure comprehensive and optimal clinical and home care of PD patients.

The data presented here does not explore other potential confounding factors, including anticholinesterase medication use, family history of dementia, comorbidities (including other neurological conditions) and their treatments, as well as gastrointestinal interacting medications, and are identified as limitations of this study. The anticholinergic drug burden could not be effectively calculated for either group, although several important known, as well as new, insights to cognition related differences in PD patients and their caregivers were identified. The lack of an unrelated control group with no association to $\mathrm{PD}$ patients may have overestimated the prevalence of cognitive impairment in the caregiver cohort, due to potential caregiver related stress caring for their spouse/family member. The results presented here should be interpreted with consideration for the limitations of the study, including selfreporting data collection, cross-sectional survey design and a potential over-representation of $\mathrm{PD}$ patients with cognitive impairment arising from recruitment at a single specialist PD clinic in metropolitan Sydney. The presented correlations and modelling were exploratory in nature and should be interpreted with caution. The lack of quantification of how much time caregivers spend with the PD patients was not ascertained in our study, which could reflect important implications for both the PD patient and caregiver's responses, as was the lack of a comprehensive clinical assessment of the caregivers, although data pertaining to their quality of life, gastrointestinal and depression characteristics was presented in earlier studies $(16,36,58)$. Another limitation includes a small proportion of the PD cohort reporting former neuroleptic medication use, as well as any head trauma, although no statistical difference in cognitive outcomes between the two groups was seen when controlling for these factors. Further limitations include the potential confounding effects of depression severity and patient disease severity rather than disease duration when investigating treatment influences, in addition to the reliance on a single cognition screening tool, the MoCA, which may miss a large segment of single domain executive dysfunction or visuospatial dysfunction, as can been seen in PDD or Dementia with Lewy Bodies, as well as having variable sensitivity and specificity. Accordingly, a more comprehensive battery of neuropsychological assessments would be useful for characterising the full range of cognitive deficits in PD and is likely to be a more sensitive index for assessing cognitive impairment (19). Likewise, appraisal with the caregiver burden scale would be more informative in the future to explore extended impacts associated from caregiver MCI. Future studies should also assess differences in the impacts of caregiver related MCI to the care of the PD patient, by characterising if they were the sole caregiver or had other opportunities to receive support for managing complex health, financial assistance, as well as emotional well-being from other caregivers.

\section{CONCLUSION}

The new insights from this study highlight the degree of MCI in PD caregivers, as well as evaluating features beyond the PD-specific factors examined in earlier studies, namely gastrointestinal dysfunction symptoms, chronic pain and physical exercise associations with PD cognitive impairment, that may positively change clinical practise. We recommend routinely screening for cognitive impairment in $\mathrm{PD}$ patients and suggest targeting constipation, chronic pain and promoting physical activity as part of a comprehensive model of care, which integrates multidisciplinary team involvement to achieve optimal PD patient and caregiver outcomes.

\section{DATA AVAILABILITY STATEMENT}

The data supporting the conclusions of this article can be made available by the authors upon request.

\section{ETHICS STATEMENT}

The studies involving human participants were reviewed and approved by the Northern Sydney Local Health District Human Research Ethics Committee and the North Shore Private Hospital Ethics Committee, HREC/18/HAWKE/109, NSPHEC 2018-LNR-009, respectively. The patients/participants provided their written informed consent to participate in this study. 


\section{AUTHOR CONTRIBUTIONS}

ML: study design, reviewed patients, collected and analysed data, drafted, and reviewed the manuscript. RD: study design, drafted, and reviewed the manuscript. CS: study design, drafted, and reviewed the manuscript. All authors contributed to the article and approved the submitted version.

\section{FUNDING}

This study was completed at the Royal North Shore Hospital in Sydney Australia. It was not industry sponsored, however support was provided by a Parkinson's New South Wales,

\section{REFERENCES}

1. Aarsland D, Bronnick K, Ehrt U, De Deyn PP, Tekin S, Emre M, et al. Neuropsychiatric symptoms in patients with Parkinson's disease and dementia: frequency, profile and associated care giver stress. J Neurol Neurosurg Psychiatry. (2007) 78:36-42. doi: 10.1136/jnnp.2005.083113

2. Lubomski M, Rushworth RL, Tisch S. Hospitalisation and comorbidities in Parkinson's disease: a large Australian retrospective study. J Neurol Neurosurg Psychiatry. (2015) 86:324-30. doi: 10.1136/jnnp-2014-307822

3. Hely MA, Reid WG, Adena MA, Halliday GM, Morris JG. The Sydney multicenter study of Parkinson's disease: the inevitability of dementia at 20 years. Mov Disord. (2008) 23:837-44. doi: 10.1002/mds.21956

4. Petersen RC, Smith GE, Waring SC, Ivnik RJ, Tangalos EG, Kokmen E. Mild cognitive impairment: clinical characterization and outcome. Arch Neurol. (1999) 56:303-8. doi: 10.1001/archneur.56.3.303

5. Hoops S, Nazem S, Siderowf AD, Duda JE, Xie SX, Stern MB, et al. Validity of the MoCA and MMSE in the detection of MCI and dementia in Parkinson disease. Neurology. (2009) 73:1738-45. doi: 10.1212/WNL.0b013e3181c34b47

6. Naismith SL, Pereira M, Shine JM, Lewis SJ. How well do caregivers detect mild cognitive change in Parkinson's disease? Mov Disord. (2011) 26:161-4. doi: $10.1002 / \mathrm{mds} .23331$

7. Naismith SL, Mowszowski L, Diamond K, Lewis SJ. Improving memory in Parkinson's disease: a healthy brain ageing cognitive training program. Mov Disord. (2013) 28:1097-103. doi: 10.1002/mds.25457

8. Wojtala J, Heber IA, Neuser P, Heller J, Kalbe E, Rehberg SP, et al. Cognitive decline in Parkinson's disease: the impact of the motor phenotype on cognition. J Neurol Neurosurg Psychiatry. (2019) 90:171-9. doi: 10.1136/jnnp-2018-319008

9. Karlstedt M, Fereshtehnejad SM, Aarsland D, Lokk J. Determinants of dyadic relationship and its psychosocial impact in patients with parkinson's disease and their spouses. Parkinsons Dis. (2017) 2017:4697052. doi: $10.1155 / 2017 / 4697052$

10. Laver K, Milte R, Dyer S, Crotty M. A systematic review and metaanalysis comparing carer focused and dyadic multicomponent interventions for carers of people with dementia. J Aging Health. (2017) 29:1308-49. doi: 10.1177/0898264316660414

11. Martinez-Martin P, Arroyo S, Rojo-Abuin JM, Rodriguez-Blazquez C, Frades B, de Pedro Cuesta J, et al. Burden, perceived health status, and mood among caregivers of Parkinson's disease patients. Mov Disord. (2008) 23:1673-80. doi: $10.1002 / \mathrm{mds} .22106$

12. Jones AJ, Kuijer RG, Livingston L, Myall D, Horne K, MacAskill $\mathrm{M}$, et al. Caregiver burden is increased in Parkinson's disease with mild cognitive impairment (PD-MCI). Transl Neurodegener. (2017) 6:17. doi: 10.1186/s40035-017-0085-5

13. Mosley PE, Moodie R, Dissanayaka N. Caregiver burden in Parkinson disease: a critical review of recent literature. J Geriatr Psychiatry Neurol. (2017) 30:235-52. doi: 10.1177/0891988717720302

14. Guo Y, Xu W, Liu FT, Li JQ, Cao XP, Tan L, et al. Modifiable risk factors for cognitive impairment in Parkinson's disease: a systematic review and
Research Seed Grant. This research was not presented at any prior meeting or conference. ML was the recipient of a RACP Research Entry and Northern Precinct Ramsay Scholarship. RD was a New South Wales Health Early-Mid Career Research Fellow. CS was a NHMRC Practitioner Fellow (APP1136800).

\section{ACKNOWLEDGMENTS}

We thank Parkinson's New South Wales for a Research Seed Grant to perform PD microbiome studies. We would also like to thank all our participants and Dr. Carly Oboudiyat for critically reviewing the manuscript. meta-analysis of prospective cohort studies. Mov Disord. (2019) 34:876-83. doi: $10.1002 / \mathrm{mds} .27665$

15. Vasconcellos LF, Pereira JS. Parkinson's disease dementia: diagnostic criteria and risk factor review. J Clin Exp Neuropsychol. (2015) 37:988-93. doi: 10.1080/13803395.2015.1073227

16. Lubomski M, Davis RL, Sue CM. Gastrointestinal dysfunction in Parkinson's disease. J Neurol. (2020) 267:1377-88. doi: 10.1007/s00415-020-0 9723-5

17. Hughes AJ, Daniel SE, Kilford L, Lees AJ. Accuracy of clinical diagnosis of idiopathic Parkinson's disease: a clinico-pathological study of 100 cases. J Neurol Neurosurg Psychiatry. (1992) 55:181-4. doi: 10.1136/jnnp.55.3.181

18. Nasreddine ZS, Phillips NA, Bedirian V, Charbonneau S, Whitehead V, Collin I, et al. The montreal cognitive assessment, MoCA: a brief screening tool for mild cognitive impairment. J Am Geriatr Soc. (2005) 53:695-9. doi: 10.1111/j.1532-5415.2005.53221.x

19. Litvan I, Goldman JG, Troster AI, Schmand BA, Weintraub D, Petersen RC, et al. Diagnostic criteria for mild cognitive impairment in Parkinson's disease: movement disorder society task force guidelines. Mov Disord. (2012) 27:349-56. doi: 10.1002/mds.24893

20. Dalrymple-Alford JC, MacAskill MR, Nakas CT, Livingston L, Graham C, Crucian GP, et al. The MoCA: well-suited screen for cognitive impairment in Parkinson disease. Neurology. (2010) 75:1717-25. doi: 10.1212/WNL.0b013e3181fc29c9

21. Goldman JG, Holden S, Ouyang B, Bernard B, Goetz CG, Stebbins GT. Diagnosing PD-MCI by MDS task force criteria: how many and which neuropsychological tests? Mov Disord. (2015) 30:402-6. doi: $10.1002 / \mathrm{mds} .26084$

22. Ware JE, Jr., Sherbourne CD. The MOS 36-item short-form health survey (SF36). I. Conceptual framework and item selection. Med Care. (1992) 30:473-83.

23. Jenkinson C, Fitzpatrick R, Peto V, Greenhall R, Hyman N. The Parkinson's disease questionnaire (PDQ-39): development and validation of a Parkinson's disease summary index score. Age Ageing. (1997) 26:353-7. doi: 10.1093/ageing/26.5.353

24. Beck AT, Ward CH, Mendelson M, Mock J, Erbaugh J. An inventory for measuring depression. Arch Gen Psychiatry. (1961) 4:561-71. doi: 10.1001/archpsyc.1961.01710120031004

25. Visser M, Leentjens AF, Marinus J, Stiggelbout AM, van Hilten JJ. Reliability and validity of the Beck depression inventory in patients with Parkinson's disease. Mov Disord. (2006) 21:668-72. doi: 10.1002/mds.20792

26. Schrag A, Barone P, Brown RG, Leentjens AF, McDonald WM, Starkstein $\mathrm{S}$, et al. Depression rating scales in Parkinson's disease: critique and recommendations. Mov Disord. (2007) 22:1077-92. doi: 10.1002/mds.21333

27. Moayyedi P, Duffett S, Braunholtz D, Mason S, Richards ID, Dowell AC, et al. The leeds dyspepsia questionnaire: a valid tool for measuring the presence and severity of dyspepsia. Aliment Pharmacol Ther. (1998) 12:125762. doi: 10.1046/j.1365-2036.1998.00404.x

28. Sood R, Ford AC. Diagnosis: Rome IV criteria for FGIDs - an improvement or more of the same? Nat Rev Gastroenterol Hepatol. (2016) 13:501-2. doi: $10.1038 /$ nrgastro.2016.110 
29. Agachan F, Chen T, Pfeifer J, Reissman P, Wexner SD. A constipation scoring system to simplify evaluation and management of constipated patients. Dis Colon Rectum. (1996) 39:681-5. doi: 10.1007/BF02056950

30. Hagstromer M, Oja P, Sjostrom M. The International Physical Activity Questionnaire (IPAQ): a study of concurrent and construct validity. Public Health Nutr. (2006) 9:755-62. doi: 10.1079/PHN2005898

31. Chaudhuri KR, Martinez-Martin P, Brown RG, Sethi K, Stocchi F, Odin $\mathrm{P}$, et al. The metric properties of a novel non-motor symptoms scale for Parkinson's disease: results from an international pilot study. Mov Disord. (2007) 22:1901-11. doi: 10.1002/mds.21596

32. Goetz CG, Tilley BC, Shaftman SR, Stebbins GT, Fahn S, MartinezMartin P, et al. Movement Disorder Society-sponsored revision of the Unified Parkinson's Disease Rating Scale (MDS-UPDRS): scale presentation and clinimetric testing results. Mov Disord. (2008) 23:2129-70. doi: $10.1002 / \mathrm{mds} .22340$

33. Stebbins GT, Goetz CG, Burn DJ, Jankovic J, Khoo TK, Tilley BC. How to identify tremor dominant and postural instability/gait difficulty groups with the movement disorder society unified Parkinson's disease rating scale: comparison with the unified Parkinson's disease rating scale. Mov Disord. (2013) 28:668-70. doi: 10.1002/mds.25383

34. Tomlinson CL, Stowe R, Patel S, Rick C, Gray R, Clarke CE. Systematic review of levodopa dose equivalency reporting in Parkinson's disease. Mov Disord. (2010) 25:2649-53. doi: 10.1002/mds.23429

35. Diagnostic and Statistical Manual of Mental Disorders: Diagnostic and Statistical Manual of Mental Disorders. 5th ed. Arlington, VA: American Psychiatric Association (2013).

36. Lubomski M, Davis RL, Sue CM. Depression in Parkinson's disease: Perspectives from an Australian cohort. J Affect Disord. (2020) 277:1038-44. doi: 10.1016/j.jad.2020.09.032

37. Augustine EF, Perez A, Dhall R, Umeh CC, Videnovic A, Cambi F, et al. Sex differences in clinical features of early, treated Parkinson's disease. PLoS ONE. (2015) 10:e0133002. doi: 10.1371/journal.pone.0133002

38. Heller J, Dogan I, Schulz JB, Reetz K. Evidence for gender differences in cognition, emotion and quality of life in Parkinson's disease? Aging Dis. (2014) 5:63-75. doi: 10.14366/AD.2014.050063

39. Lubomski M, Louise Rushworth R, Lee W, Bertram KL, Williams DR. Sex differences in Parkinson's disease. J Clin Neurosci. (2014) 21:1503-6. doi: 10.1016/j.jocn.2013.12.016

40. Kierzynka A, Kazmierski R, Kozubski W. Educational level and cognitive impairment in patients with Parkinson disease. Neurol Neurochir Pol. (2011) 45:24-31. doi: 10.1016/S0028-3843(14)60056-6

41. Lin YQ, Chen SD. RBD: a red flag for cognitive impairment in Parkinson's disease? Sleep Med. (2018) 44:38-44. doi: 10.1016/j.sleep.2018.01.006

42. Braak H, Del Tredici K, Rub U, de Vos RA, Jansen Steur EN, Braak E. Staging of brain pathology related to sporadic Parkinson's disease. Neurobiol Aging. (2003) 24:197-211. doi: 10.1016/S0197-4580(02)00065-9

43. Ehrt U, Broich K, Larsen JP, Ballard C, Aarsland D. Use of drugs with anticholinergic effect and impact on cognition in Parkinson's disease: a cohort study. J Neurol Neurosurg Psychiatry. (2010) 81:160-5. doi: 10.1136/jnnp.2009.186239

44. Yarnall AJ, Lawson RA, Duncan GW, Breen DP, Khoo TK, Brooks D, et al. Anticholinergic load: is there a cognitive cost in early Parkinson's disease? J Parkinsons Dis. (2015) 5:743-7. doi: 10.3233/JPD-150664

45. Wenzel K, Homann CN, Fabbrini G, Colosimo C. The role of subcutaneous infusion of apomorphine in Parkinson's disease. Expert Rev Neurother. (2014) 14:833-43. doi: 10.1586/14737175.2014.928202

46. Lawson RA, Yarnall AJ, Duncan GW, Khoo TK, Breen DP, Barker RA, et al. Severity of mild cognitive impairment in early Parkinson's disease contributes to poorer quality of life. Parkinsonism Relat Disord. (2014) 20:1071-5. doi: 10.1016/j.parkreldis.2014.07.004

47. Lawson RA, Yarnall AJ, Duncan GW, Breen DP, Khoo TK, Williams-Gray $\mathrm{CH}$, et al. Cognitive decline and quality of life in incident Parkinson's disease: the role of attention. Parkinsonism Relat Disord. (2016) 27:47-53. doi: 10.1016/j.parkreldis.2016.04.009
48. Lawson RA, Collerton D, Taylor JP, Burn DJ, Brittain KR. Coping with cognitive impairment in people with Parkinson's disease and their carers: a qualitative study. Parkinsons Dis. (2018) 2018:1362053. doi: $10.1155 / 2018 / 1362053$

49. Schrag A, Jahanshahi M, Quinn NP. What contributes to depression in Parkinson's disease? Psychol Med. (2001) 31:65-73. doi: 10.1017/s0033291799003141

50. Uc EY, McDermott MP, Marder KS, Anderson SW, Litvan I, Como $\mathrm{PG}$, et al. Incidence of and risk factors for cognitive impairment in an early Parkinson disease clinical trial cohort. Neurology. (2009) 73:1469-77. doi: 10.1212/WNL.0b013e3181bf992f

51. Lubomski M, Tan AH, Lim SY, Holmes AJ, Davis RL, Sue CM. Parkinson's disease and the gastrointestinal microbiome. J Neurol. (2020) 267:2507-23. doi: 10.1007/s00415-019-09320-1

52. Lubomski M, Davis RL, Sue CM. The gut microbiota: a novel therapeutic target in Parkinson's disease? Parkinsonism Relat Disord. (2019) 66:265-6. doi: 10.1016/j.parkreldis.2019.08.010

53. Murray DK, Sacheli MA, Eng JJ, Stoessl AJ. The effects of exercise on cognition in Parkinson's disease: a systematic review. Transl Neurodegener. (2014) 3:5. doi: 10.1186/2047-9158-3-5

54. Goodarzi Z, Ismail Z. A practical approach to detection and treatment of depression in Parkinson disease and dementia. Neurol Clin Pract. (2017) 7:128-40. doi: 10.1212/CPJ.00000000000 00351

55. Skogar O, Lokk J. Pain management in patients with Parkinson's disease: challenges and solutions. J Multidiscip Healthc. (2016) 9:469-79. doi: 10.2147/JMDH.S105857

56. Goldman JG, Vernaleo BA, Camicioli R, Dahodwala N, Dobkin RD, Ellis T, et al. Cognitive impairment in Parkinson's disease: a report from a multidisciplinary symposium on unmet needs and future directions to maintain cognitive health. NPJ Parkinsons Dis. (2018) 4:19. doi: 10.1038/s41531-018-0055-3

57. Cooper LA, Marsteller JA, Carson KA, Dietz KB, Boonyasai RT, Alvarez $\mathrm{C}$, et al. The RICH LIFE project: a cluster randomized pragmatic trial comparing the effectiveness of health system only vs. health system Plus a collaborative/stepped care intervention to reduce hypertension disparities. Am Heart J. (2020) 226:94-113. doi: 10.1016/j.ahj.2020. 05.001

58. Lubomski M, Davis RL, Sue CM. Health-related quality of life in Parkinson's disease patients and their caregivers. J Mov Disord. (2021) 14:42-52. doi: 10.14802/jmd.20079

59. Palavra NC, Lubomski M, Flood VM, Davis RL, Sue CM. Increased added sugar consumption is common in Parkinson's disease. Front Nutr. (2021) 8:207. doi: $10.3389 /$ fnut.2021.628845

60. Bhimani R. Understanding the burden on caregivers of people with Parkinson's: a scoping review of the literature. Rehabil Res Pract. (2014) 2014:718527. doi: $10.1155 / 2014 / 718527$

Conflict of Interest: The authors declare that the research was conducted in the absence of any commercial or financial relationships that could be construed as a potential conflict of interest.

Publisher's Note: All claims expressed in this article are solely those of the authors and do not necessarily represent those of their affiliated organizations, or those of the publisher, the editors and the reviewers. Any product that may be evaluated in this article, or claim that may be made by its manufacturer, is not guaranteed or endorsed by the publisher.

Copyright (C) 2021 Lubomski, Davis and Sue. This is an open-access article distributed under the terms of the Creative Commons Attribution License (CC BY). The use, distribution or reproduction in other forums is permitted, provided the original author(s) and the copyright owner(s) are credited and that the original publication in this journal is cited, in accordance with accepted academic practice. No use, distribution or reproduction is permitted which does not comply with these terms. 